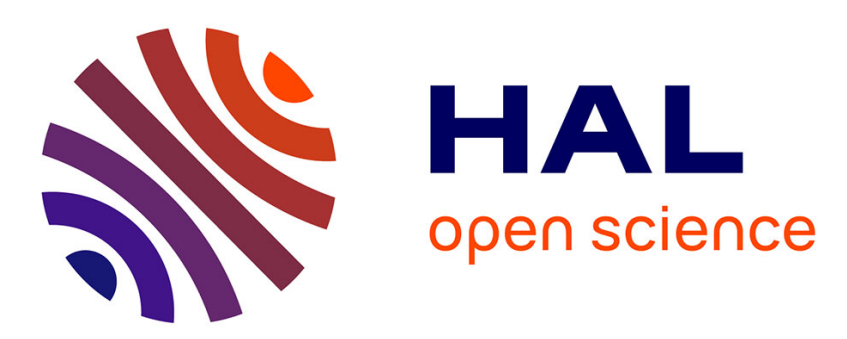

\title{
Identification externe du sexe chez le saumon atlantique (Salmo salar L.)
}

Gérard Maisse, Jean-Luc J.-L. Baglinière, G. Landry, F. Caron, A. Rouleau

\section{To cite this version:}

Gérard Maisse, Jean-Luc J.-L. Baglinière, G. Landry, F. Caron, A. Rouleau. Identification externe du sexe chez le saumon atlantique (Salmo salar L.). Canadian Journal of Zoology, 1988, 66, pp.2312-2315. 10.1139/z88-343 . hal-02728159

\section{HAL Id: hal-02728159 \\ https://hal.inrae.fr/hal-02728159}

Submitted on 2 Jun 2020

HAL is a multi-disciplinary open access archive for the deposit and dissemination of scientific research documents, whether they are published or not. The documents may come from teaching and research institutions in France or abroad, or from public or private research centers.
L'archive ouverte pluridisciplinaire HAL, est destinée au dépôt et à la diffusion de documents scientifiques de niveau recherche, publiés ou non, émanant des établissements d'enseignement et de recherche français ou étrangers, des laboratoires publics ou privés. 


\title{
Identification externe du sexe chez le Saumon atlantique (Salmo salar L.)
}

\author{
G. Maisse et J. L. Baglinitèn \\ Institut national de la recherche agronomique, Station de physiologie et écologie des poissons, \\ 35042 Rennes CEDEX, France
}

ET

G. Landry, F. Caron et A. Rouleau

Ministère du Loisir de la Chasse et de la Pêche du Québec, 150, boulevard Saint-Cyrille est, Québec (Québec), Canada

Reçu le 12 juin, 1987

Maisse, G., Baglinière, J. L., Landry, G., Caron, F., et Rouleau, A. 1988. Identification externe du sexe chez le Saumon atlantique (Salmo salar L.). Can. J. Zool. 66: 2312-2315.

Une technique d'identification exteme du sexe chez le Saumon atlantique (Salmo salar) est proposée. Deux cent quatorze individus capturés en juillet et août 1986 dans cinq rivières québécoises et à un site en eau salée ont été étudiés. Pour l'ensemble de l'échantillon, le dimorphisme sexuel de la longueur relative de la mâchoire supérieure a permis une identification exacte du sexe dans $88 \%$ des cas.

Maisse, G., Baglinière, J. L., Landry, G., Caron, F., and Rouleau, A. 1988. Identification externe du sexe chez le Saumon atlantique (Salmo salar L.). Can. J. Zool. 66: 2312-2315.

A technique to determine the sex of the atlantic salmon (Salmo salar) from external features is proposed. A total of 214 individuals caught in July and August 1986 in five rivers in Quebec and at a salt waterlocation have been studied. Overall, the sexual dimorphism of the relative length of the upper jaw bone allowed us to sex the fishes with $88 \%$ success.

\section{Introduction}

Une des recommandations de l'atelier sur l'âge à maturité des Salmonidés, qui s'est tenu à la station biologique de St. Andrews en 1984 , a été la mise en place du suivi de plusieurs rivières pilotes pour lesquelles les caractéristiques des populations exploitées et inexploitées pouvaient être recueillies annuellement (Porter et al. 1986). Il va de soi que la réussite d'un tel suivi dépend directement de la qualité des données recueillies; l'une des plus difficilement accessibles dans l'étude des géniteurs de Saumon atlantique est le sexe, avant l'apparition du bec caractérisant le mâle en période de fraie. Au printemps et en été, seule l'autopsie des poissons pêchés donne une information sûre. Pour les géniteurs gardés vivants, Le Bail et Breton (1981) ont mis au point une technique de détection des femelles en vitellogenèse par mise en évidence de la vitellogénine plasmatique par séroagglutination. Cette technique, bien qu'adaptée aux conditions de terrain par Le Bail et al. (1981), est d'un usage réservé à un personnel initié. Maisse et Baglinière (1986) s'inspirant des résultats obtenus par Le Bail (1981) sur la Truite de mè (Salmo trutta) ont mis en évidence la possibilité d'identifier le sexe des adultes de Saumon atlantique (Salmo salar) de la rivière North Esk (Écosse) à partir de la longueur relative de la mâchoire supérieure. La fiabilité, moins de $10 \%$ d'erreur, et la facilité de mise en oeuvre de cette technique nous ont amené à tester celle-ci sur les captures estivales de Saumon atlantique dans quelques rivières québécoises.

\section{Matériel et méthodes}

Les 214 saumons étudiés provenaient des captures par pêche à la ligne réalisées sur les rivières Moisie $(n=72)$, Jupiter $(n=48)$, Bonaventure $(n=49)$ et Grande Cascapédia $(n=30)$ (fig. 1$)$ et des pêches commerciales $(n=15)$ en juillet 1986. Cent quatre-vingt-neuf saumons capturés à la ligne de juin à août 1986 sur la rivière de la Trinité ont été étudiés parallèlement.

La longueur de la mâchoire supérieure (LM, en millimètres) selon Le Bail (1981) (fig. 2) et la longueur à la fourche (LF, en millimètres, précision de $5 \mathrm{~mm}$ ) ont été mesurées pour chaque poisson dont le sexe était déterminé après autopsie.

Pour chaque individu, des écailles ont été prélevées et l'âge de mer ainsi que les marques de fraie ont été déterminés selon les normes du Conseil international pour l'exploration de la mer (Anonyme 1984).

Le traitement des données a été réalisé par analyse factorielle discriminante (Lefêbvre 1976).

\section{Résultats}

Quelle que soit la rivière, le dimorphisme sexuel de la longueur relative de la mâchoire supérieure apparaît clairement : à taille égale, le mâle possède une mâchoire supérieure plus longue que celle de la femelle (fig. 3 ).

En raison d'un rapport des sexes très déséquilibré en faveur des mâles chez les madeleineaux (un hiver marin, LF = $630 \mathrm{~mm}$ ), nous n'avons effectué l'analyses factorielle discriminante que chez les saumons rédibermarins (plusieurs hivers marins). Les résultats de cette analyse sont donnés dans le tableau 1. La discrimination est très hautement significative avec $\chi^{2}=156,3$ pour $D L=2$.

L'application de l'équation discriminante aux données initiales indique que 22 poissons sur 182 sont mal classés (12 mâles et 10 femelles) soit $12 \%$. Il n'y a pas de différence significative entre les proportions d'individus mal classés calculées pour chaque rivière (test de $\chi^{2}$ ).

\section{Discussion et conclusion}

Les résultats de cette étude confirment la possibilité de connaître le sexe du Saumon atlantique à partir du dimorphisme 


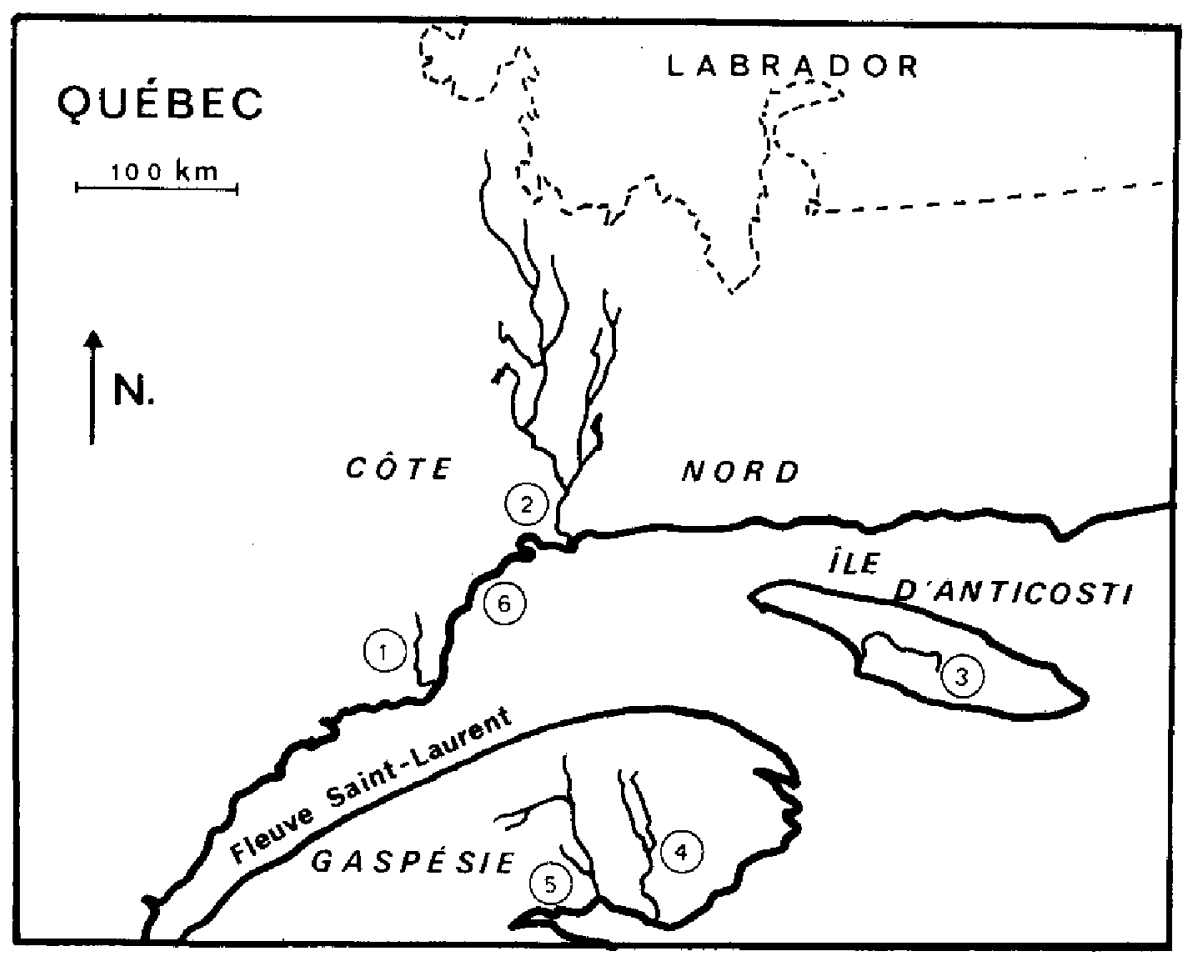

FIG. 1. Situation des rivières échantillonnées au Québec. 1, rivière de la Trinité; 2, rivière Moisie; 3, rivière Jupiter; 4, rivière Bonaventure; 5 , rivière Cascapédia; 6 , site en eau salée.

Tableau 1. Résultats de l'analyse factorielle discriminante chez les saumons rédibermarins $(n=182)$

\begin{tabular}{ll}
\hline \hline Équation discriminante & \\
$\quad(y<0$ pour les mâles et $y>0$ pour les femelles) & $Y=-0,2866 \mathrm{LM}+0,0324 \mathrm{LF}-4,0262$ \\
Droite de séparation & $\mathrm{LM}=0,1130 \mathrm{LF}-14,0482$ \\
\hline
\end{tabular}

Nota : LF, longueur à la fourchẹ; LM, longueur de la mâchoire supérieure.

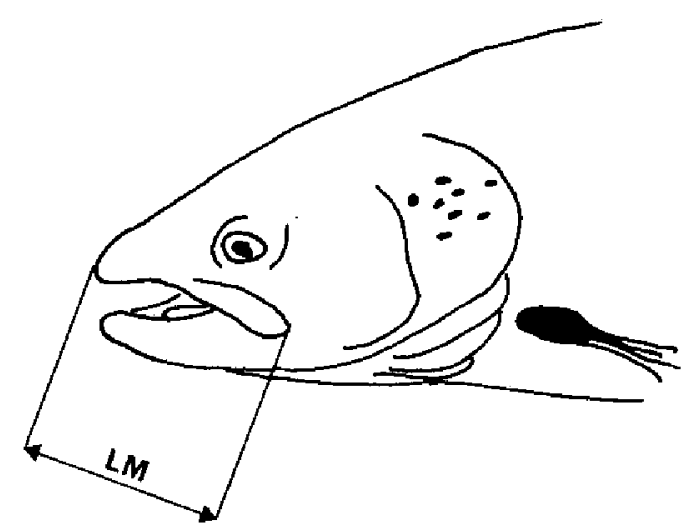

FIG. 2. Longueur de la mâchoire supérieure (LM) (mesure effectuée à l'aide d'un compas à pointes sèches).

sexuel de la longueur relative de la mâchoire supérieure. La faibilité de cette méthode dépend directement de l'état physiologique des individus capturés. En effet, les modifications morphologiques caractéristiques des mâles devenant matures sont sous dépendance androgénique (Liley et Stacey 1983); Idler et al. (1971) ont décrit l'évolution des niveaux d'androgènes plasmatiques chez les mâles de Saumons atlantiques capturés dans la rivière Miramichi (Nouveau-Brunswick). Il est probable que les niveaux faibles, rapportés par ces auteurs chez les individus capturés fin août, se rencontrent à la même époque chez les mâles capturés au Québec. Ceci peut étre
TABleau 2. Nombre, selon le sexe et l'âge de mer à la première remontée, des saumons capturés dans la rivière de la Trinité au cours de l'été 1985

\begin{tabular}{lcc}
\hline & Madeleineaux & Rédibermarins \\
\hline Mâles & 77 & 5 \\
Femelles & 2 & 105 \\
\hline
\end{tabular}

l'explication du taux d'erreur de $12 \%$, qui devrait diminuer en automne, comme l'a montré Richard (1986) chez la Truite de mer (10\% d'erreur au printemps; $2 \%$, en automne).

Ce caractère sexuel secondaire apparait chez de nombreux salmonidés : la Truite commune (Salmo trutta) (Le Bail 1981), le Saumon coho (Oncorhynchus kisutch) (Le Bail 1981; Beacham et Murray 1986), la Truite de mer (Le Bail 1981; Richard 1986), l'Omble chevalier (Salvelinus alpinus) (G. Maisse et B. Breton, données non publiées) et le Saumon atlantique (Maisse et Baglinière 1986). Dans le cas de la rivière de la Trinité, le rapport des sexes est déséquilibré à la fois chez les madeleineaux et chez les saumons rédibermarins (tableau 2), l'identification du sexe pouvant se faire sur l'âge de mer à la première remontée et les madeleineaux étant alors en très grande majorité des mâles et les rédibermarins des femelles. L'application de l'équation discriminante à ce stock permet l'identification correcte du sexe de 56 des 60 rédibermarins mesurés. 


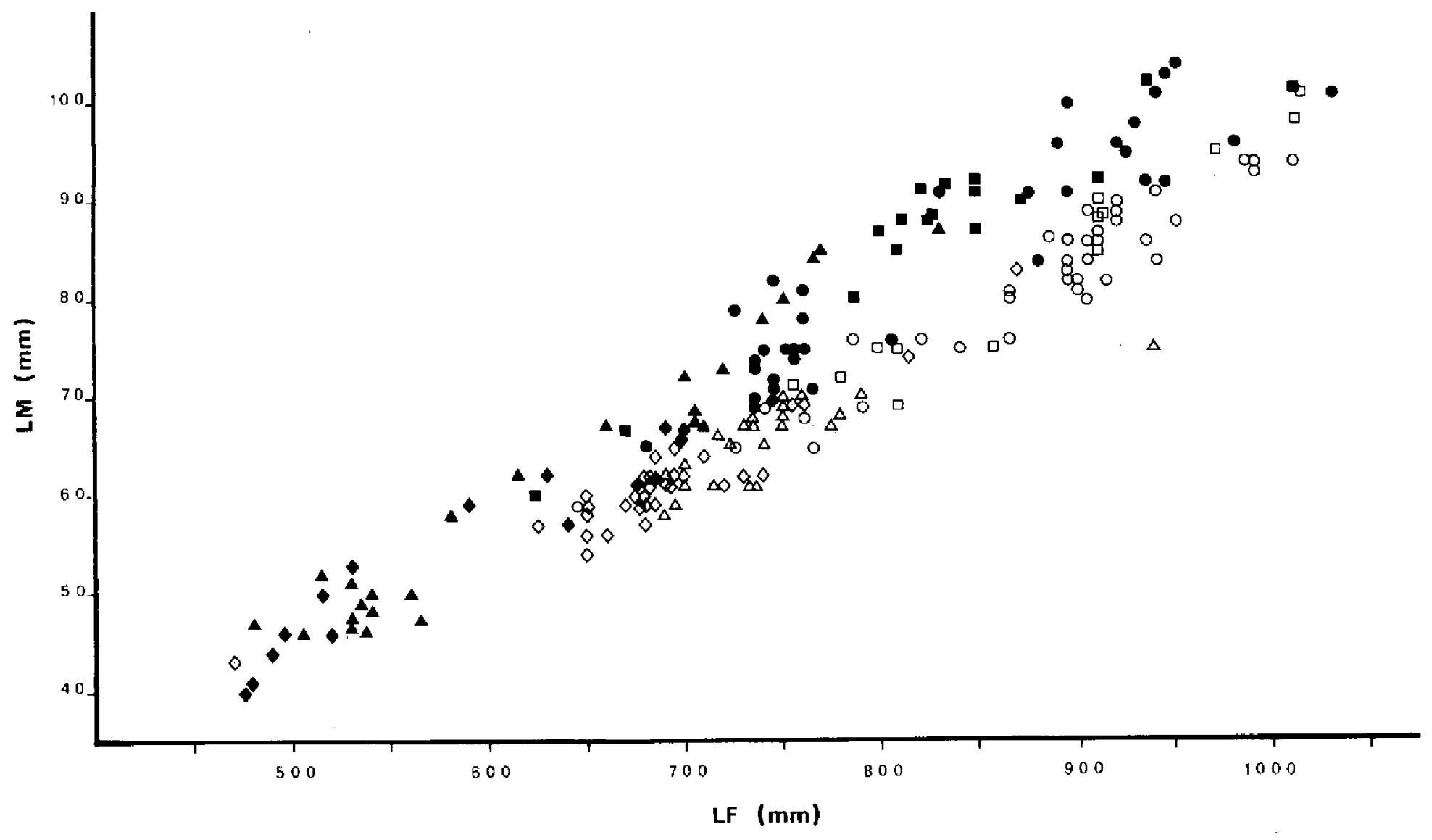

FIG. 3. Relation entre la longueur de la mâchoire supérieure (LM) et la longueur à la fourche (LF) selon le sexe ( $\sigma^{*}$, symboles noirs; $\$$, symboles blancs) et la rivière. $O$ et $O$, rivière Moisie; $\boldsymbol{Q}$ et $\square$, rivière Cascapédia; $\boldsymbol{\Delta}$ et $\Delta$, rivière Bonaventure; et $\diamond$, rivière Jupiter.

TABleau 3. Comparaison des fiabilités des différentes techniques d'identification du sexe chez le Saumon atlantique

\begin{tabular}{|c|c|c|}
\hline Technique & $\begin{array}{c}\text { Succès } \\
(\%)\end{array}$ & Références \\
\hline Empirisme, allure générale & 78 & Maisse et Baglinière (1986) \\
\hline Dimorphisme de la nageoire adipeuse & 75 & Maisse et Baglinière (1986) \\
\hline Dimorphisme de la machoire supérieure & $88-92$ & $\begin{array}{l}\text { Maisse et Balinière (1986); } \\
\text { cette étude }\end{array}$ \\
\hline Mesure de la 11-cétotestostérone plasmatique & 96 & Le Bail et al. (1983) \\
\hline $\begin{array}{l}\text { Immunoagglutination de la vitellogénine } \\
\text { plasmatique }\end{array}$ & 100 & $\begin{array}{l}\text { Le Bail et Breton (1981); } \\
\text { Maisse et Baglinière (1986) }\end{array}$ \\
\hline
\end{tabular}

La technique que nous avons testée est simple, avec deux mesures de longueur à effectuer, et sa fiabilité, avec environ $90 \%$ de succès, est comparable à celle des autres techniques employées dans l'étude des populations naturelles de Salmonidés (scalimétrie, estimation des densités, piégeage ... ).

Comparée aux autres méthodes d'identification du sexe appliquées au Saumon atlantique (tableau 3), elle est certe moins précise que les analyses sanguines, mais tellement plus simple à mettre en oeuvre que nous pensons quelle peut être facilement et rapidement incluse dans les programmes d'étude des géniteurs migrants de Saumon atlantique.

\section{Remerciements}

La collecte des données a été rendue possible grâce à la collaboration des pêcheurs sportifs du club de pêche au saumon Moisie, de la Pourvoirie de la Société d'établissement des parcs et de plein air du Québec sur la rivière Jupiter, de la zone d'exploitation contrôlée et du club de pêche au saumon de Bonaventure, de la Société Cascapédia inc. de la zone d'exploitation contrôlée de Baie-Trinité sur la rivière de la Trinité et de Monsieur Lucien Lebel sur la Côte-Nord du SaintLaurent. Monsieur Y. Côté (ministère du Loisir de la Chasse et de la Pêche du Québec) a contribué grandement à l'organisation et à la réussite de la campagne d'échantillonnage.

ANONYme. 1984. Atlantic salmon scale reading. Report of Atlantic Salmon Scale Reading Workshop, Aberdeen, 23-28 avril 1984. International Council for the Exploration of the Sea.

Beacham, T. D., et Murray, C. B. 1986. Sexual dimorphism in length of upper jaw and adipose fin of immature and maturing Pacific salmon (Oncorhynchus). Aquaculture, 58 : 269-276.

IdLeR, D. R., Horne, D. A.; et SANGALANG, G. B. 1971. Identification and quantification of the major androgens in testicular and 
peripheral plasma of atlantic salmon (Salmo salar) during sexual maturation. Gen. Comp. Endocrinol. $16: 257-267$.

LE BaIL, P. Y. 1981. Identification du sexe en fonction de l'état de maturité chez les poissons. Thèse de docteur ingénieur, École nationale supérieure d'agronomie, Rennes.

Le BAIL, P. Y., et BRETON, B. 1981. Rapid determination of the sex of puberal salmonid fish by a technique of immunoagglutination. Aquaculture, $22: 367-375$.

Le Ball, P. Y., Maisse, G., et Breton, B. 1981. Détection des femelles de salmonidés en vitellogenèse. 1. Description de la méthode et mise en oeuvre pratique. Bull. Fr. Piscic. 283 : 79-88.

Le Bail, P.-Y., Fostigr, A., et Marcuzzi, O. 1983. Limites et améliorations du sexage des salmonidés par dosage de la 11-cétotestostérone circulante. Can. J. Zool. 61 : 457-460.

LefÈbvRE, J. 1976. Introduction aux analyses statistiques multidimentionnelles. Masson, Paris.
Liley, N. R, et Stacey, N. E. 1983. Hormones, pheromones and reproductive behavior in fish. Dans Fish physiology. Vol. IX, partie B. Editeurs : W. S. Hoar, D. J. Randall et E. M. Donaldson. Academic Press, New York. pp. 1-63.

Maisse, G., et Baglinière, J. L. 1986. Note technique : le sexage morphologique du saumon atlantique (Salmo salar). Bull. Fr. Peche Piscic. $300: 1-12$.

Porter, T. R., Healey, M. C., et O'Connell, M. F. 1986. Implications of varying the sea age at maturity of atlantic salmon (Salmo salar) on yield to the fisheries. Dans Salmonid age at maturity. Éditeur : D. J. Meerburg. Can. Spec. Publ. Fish. Aquat. Sci. 89 : 110-117.

RichaRD, A. 1986. Recherches sur la truite de mer, Salmo trutta L., en Basse-Normandie. Thèse de $3^{\mathrm{e}}$ cycle, Unité d'enseignement et de recherche en Sciences de la vie et de l'environnement, Université de Rennes I, Rennes. 